\title{
Análise da estimativa da evapotranspiração por diferentes modelos para Amparo de São
}

\section{Francisco - Sergipe}

\author{
Analysis of evapotranspiration estimation by different models for Amparo de São Francisco - Sergipe
}

Análisis de estimación de evapotranspiración por diferentes modelos para Amparo de São Francisco Sergipe

\section{Resumo}

É de fundamental importância conscientizar a população sobre o uso dos recursos hídricos, expondo cada vez mais a sociedade que os mesmos dependem de um período de regeneração e que o uso irresponsável vem a provocar escassez d'água. Tem-se como objetivo avaliar e comparar o desempenho de métodos empíricos de estimativa da evapotranspiração pelos métodos de Hargreaves, Hargreaves-Samani, Camargo e Thorntwaite com o método padrão de Penman-Monteih, sugerido pela FAO-56, para Amparo de São Francisco - Sergipe. Os dados pluviais foram do período 1963-2020 e os dados térmicos foram estimados pelo software estima - T. Os dados climatológicos foram estimados pelo método da interpolação simplificada. Com os resultados, ficou claro que as mudanças de uso da terra, tais como agricultura, pastagem e reflorestamento causam alterações na dinâmica da água de uma bacia hidrográfica. $\mathrm{O}$ método de Penman-Monteith apontado pela FAO para a determinação da ETp, apresentam valores diferentes das reais condições hídricas, pelo menos na área estudada, superestimando a ETp mensal em todos os usos da terra estudados. Este comportamento pode estar atrelado a radiação solar e a temperatura que são os fatores determinantes para a estimativa da evapotranspiração pelo método de Penman-Monteith.

Palavras-chave: Dados climatológicos; Diferentes métodos ETo; Manejo de irrigação.

\begin{abstract}
It is of fundamental importance to make the population aware of the use of water resources, increasingly exposing society that they depend on a period of regeneration and that irresponsible use causes water shortages. The objective is to evaluate and compare the performance of empirical methods for estimating evapotranspiration by the methods of Hargreaves, Hargreaves-Samani, Camargo and Thorntwaite with the standard method of Penman-Monteih, suggested by FAO-56, for Amparo de São Francisco - Sergipe. The rainfall data were from the period 1963-2020 and the thermal data were estimated by the software estima - T. The climatological data were estimated by the simplified interpolation method. With the results, it became clear that land use changes, such as agriculture, grazing and reforestation, cause changes in the water dynamics of a watershed. The Penman - Monteith method indicated by the FAO for the determination of ETp, present values different from the real water conditions, at least in the studied area, overestimating the monthly ETp in all studied land uses. This
\end{abstract}


behavior may be linked to solar radiation and temperature, which are the determining factors for the estimation of evapotranspiration by the Penman-Monteith method.

Keywords: Climatological data; Different ETo methods; Irrigation management.

\section{Resumen}

Es de fundamental importancia concienciar a la población sobre el uso de los recursos hídricos, exponiendo cada vez más a la sociedad que dependen de un período de regeneración y que el uso irresponsable provoca escasez de agua. El objetivo es evaluar y comparar el desempeño de métodos empíricos para estimar la evapotranspiración por los métodos de Hargreaves, Hargreaves-Samani, Camargo y Thorntwaite con el método estándar de Penman-Monteih, sugerido por FAO56, para Amparo de São Francisco - Sergipe. . Los datos de precipitación fueron del período 1963-2020 y los datos térmicos fueron estimados por el software estima - T. Los datos climatológicos fueron estimados por el método de interpolación simplificado. Con los resultados, quedó claro que los cambios en el uso de la tierra, como la agricultura, el pastoreo y la reforestación, provocan cambios en la dinámica del agua de una cuenca. El método Penman-Monteith indicado por la FAO para la determinación de ETp, presenta valores diferentes a las condiciones reales del agua, al menos en el área estudiada, sobrestimando la ETp mensual en todos los usos de suelo estudiados. Este comportamiento puede estar relacionado con la radiación solar y la temperatura, que son los factores determinantes para la estimación de la evapotranspiración por el método Penman-Monteith.

Palabras clave: Datos climatológicos; Diferentes métodos ETo; Manejo de riego.

\section{Introdução}

A estimativa da evapotranspiração (ETo) pode ser realizada por métodos diretos ou indiretos (Cunha, Magalhaes \& Castro 2013). Os métodos indiretos são menos onerosos, e se baseiam em fórmulas matemáticas empíricas que combinam dados climatológicos medidos em estações meteorológicas (Pereira, Villa Nova \& Sediyana 1997), dentre eles podemos citar: Thornthwaite (1948); Penman (1948); Priestley e Taylon (1972); Doorenbos e Prush (1977); Hargreaves (1977); Penman-Monteith FAO-56 (allen, Pereira, Raes e Smith 1998). Barros, Souza, Fonseca e Silva. (2009), Unlu, Kapur (2010), Schrader, Duner, Frank, Gebler, Putz, Hannes e Wollschger (2013) e Gebler, Franssem, Putz, PostSchmith e vereecken (2015). O uso de métodos baseados em equações com fundamentação física é a forma mais utilizada por pesquisadores, sendo o modelo de Penman-Monteith recomendado pela Organização das Nações Unidas para Alimentação e Agricultura (Food and Agriculture Organization - FAO). Para Goulden, Litvak e Miller (2007), as diferentes técnicas de estimativa resultam em diferentes resultados de evapotranspiração para um mesmo local.

A importância na escolha do método mais adequado para estimativa da ETo, ocorre em função das dificuldades em mensurar diretamente as perdas além do conhecimento relativo à quantidade d'água a ser aplicada em determinada atividade, de forma a se obter maior exatidão, reduzindo as perdas e custos com água e energia.

A evaporação, assim como a evapotranspiração, soma perdas d'água acentuadas para as áreas semiáridas do Brasil, sendo de grande importância à identificação dos fatores que afetam o balanço hídrico regional (Tucci, 2005). Um dos elementos climáticos de importância no estudo da economia d'água dos reservatórios é a evapotranspiração visando uma utilização de forma racional. Isto porque o conhecimento do consumo de água pelas culturas, ou seja, a evapotranspiração das culturas leva em consideração a precipitação e as perdas operacionais dos sistemas de irrigação (Sediyama, 1996).

Apesar do modelo de Penman-Monteith demonstrar resultados apurados na estimativa da ETo em diferentes condições climáticas, ele necessita de diversas variáveis meteorológicas, como radiação solar, temperatura e umidade relativa do ar, velocidade do vento, entre outras, que nem sempre estão disponíveis em todas as regiões (Santos, Lyra, Lima, Souza \& Delgado 2016). Para tanto, diferentes modelos que utilizam um menor número de variáveis têm sido comumente aplicados como alternativas ao PenmanMonteith. Porém, esses modelos podem não apresentar boas estimativas, superestimando ou subestimando a ETo quando comparados com o método padrão (Dantas, Oliveira, Dalri, Palarreti, Santos \& Farias 2016). 
Borges e Mendiondo (2007) constataram que a exatidão dos modelos de Camargo, Hargreaves, Thornthwaite na estimativa de ETo para a Bacia do rio Jacupiranga foram excelentes. Os resultados dos métodos de Hargreaves e Camargo foram mais eficientes na estimativa da ETo.

Medeiros et al. (2020) especializaram pelo método da krigagem os elementos precipitação, evapotranspiração e evaporação prováveis ao nível de 75\% de probabilidade para 187 municípios foi levada nas estações verão e outono na região litorânea, zona da Mata e Agreste, gerando suas cartas e informações para os tomadores de decisões governamentais. Os autores concluíram que nas estações primavera e inverno as contribuições pluviais foram decorrentes dos efeitos locais, orografia e sistemas de escala local ocasionando chuvas fracas a moderadas em curto intervalo de tempo. Na falta de elementos meteorológicos necessários para determinar a evapotranspiração, a equação de Thornthwaite pode ser utilizada com razoável precisão para estimativa da evapotranspiração na área estudada.

A evapotranspiração (ETP) representa a chuva teoricamente necessária para não faltar nem sobrar água no solo e é utilizada na determinação do balanço hídrico para a identificação dos períodos de excesso ou escassez de água (Camargo \& Sentelha 1999; Barros, Araújo Filho, Silva \& Santigo 2012; Costa, Medeiros e Gomes Filho 2015).

Medeiros et al. (2016), em estudos sobre a ETP no município de Campina Grande - PB observaram que a ETP anual é de 1076,8 $\mathrm{mm}$, com variações de 105,4 mm no mês de dezembro a $71,3 \mathrm{~mm}$ no mês de agosto. Este valor no mês de agosto é menor devido a característica do final do inverno no Hemisfério Sul. O estudo também relata que a influência local tem grande contribuição sobre o aquecimento pontual, o chamado "aquecimento urbano" que se pode constatar nitidamente na área em estudo.

Tem-se como objetivo avaliar o desempenho de métodos empíricos de estimativas da ETo, Hargreaves, HargreavesSamani, Camargo e Thorntwaite e comparar com o método padrão de Penman-Monteih, sugerido pela FAO-56, para Amparo de São Francisco - Sergipe

\section{Material e Métodos}

Amparo de São Francisco limita-se com Telha a Leste e a Sul, Canhoba a Oeste, o Estado de Alagoas a Norte. Com altitude de 51 metros e de coordenadas geográficas de $10^{\circ} 08^{\prime} 04^{\prime \prime}$ sul e $36^{\circ} 55^{\prime} 46^{\prime \prime}$ Oeste. (Figura 1).

Figura 1. O Nordeste de Sergipe e o posicionamento da cidade de Amparo de São Francisco.

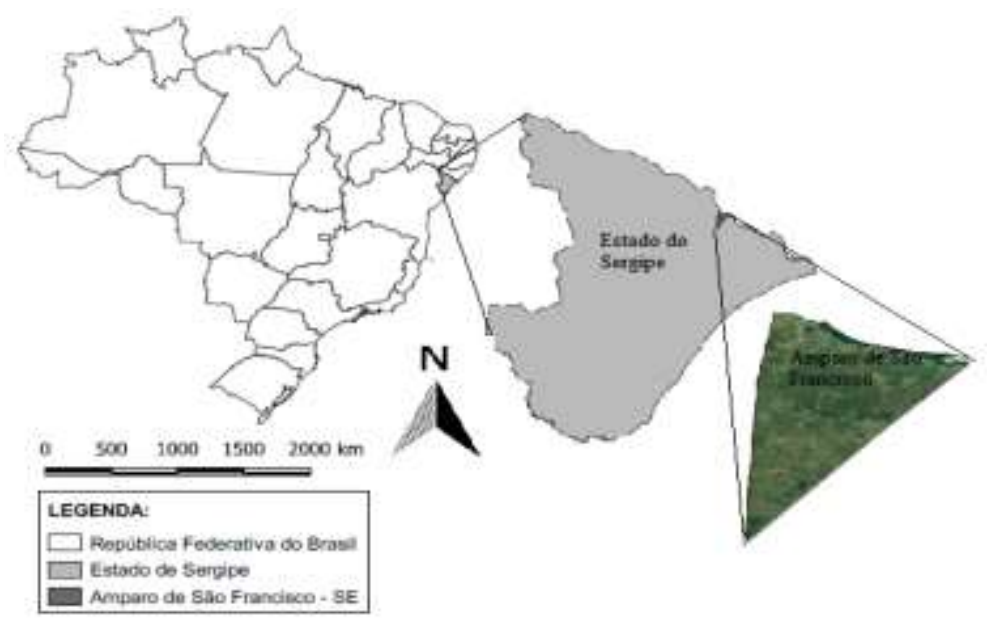

Fonte: França (2021). 
Amparo de São Francisco localiza-se em uma área marcada por duas estações bem definidas, um período chuvoso fluindo de fevereiro a agosto e seu período seco entre setembro a janeiro. Conforme a classificação climática de Köppen (1928) e Köppen e Geigom (1931), o município tem o clima do tipo “As" (quente e úmido tropical chuvoso). Os estudos dos autores (Medeiros, 2020) e (Alvares, Stape, Sentelhas, Gonçalves, \& Sparovek 2014) corroboram com os resultados encontrados. Utilizaram-se dados de precipitações mensais e anuais fornecidos pela Superintendência de Desenvolvimento do Nordeste (SUDENE, 1990); e Empresa de Desenvolvimento Agropecuário de Sergipe (ENDAGRO-SE, 2020) entre 1963 a 2019. Os dados térmicos foram estimados pelo programa computacional Estima -T (Cavalcanti, Silva \& Souza 2006; Cavalcanti \& Silva 1994). Os demais dados climatológicos foram estimados pelo método da interpolação simples (Medeiros, 2020).

\section{Modelo evapotranspirativo de Penman-Monteith}

Foi desenvolvido pela sua consistência visando avaliar a evapotranspiração $\left(\mathrm{ETo}_{(\mathrm{PM})}\right)$ em diferentes locais e climas e por considerar como parâmetros de trocas de energia e fluxo de calor latente. Este método foi definido em 1990 pela FAO-56 (Allen et al., 1998) como o método padrão para estimativa de $\left(\mathrm{ETo}_{(\mathrm{PM})}\right)$. Este método permite estimar a $\left(\mathrm{ETo}_{(\mathrm{PM})}\right)$ em mm/mês e após seu computo, multiplicou-se pelo número total de dias de cada mês do ano, obtendo-se assim o modelo do Penman-Monteith estimado em $\mathrm{mm} / \mathrm{mês}$.

$$
\mathrm{ETo}_{(\mathrm{PM}-56)}=\frac{0,408 \Delta\left(\mathrm{R}_{\mathrm{n}}-\mathrm{G}\right)+\gamma \frac{900}{\mathrm{~T}+273} \mathrm{u}_{2}\left(\mathrm{e}_{\mathrm{s}}-\mathrm{e}_{\mathrm{a}}\right)}{\Delta+\gamma\left(1+0,34 \mathrm{u}_{2}\right)}
$$

Onde:

ETo - evapotranspiração de referência, $\mathrm{mm} / \mathrm{mês}$;

$\delta$ - curva de pressão de vapor, $\left(\mathrm{kPa} /{ }^{\circ} \mathrm{C}\right)$;

$R_{n}$ - saldo da radiação mensal, (MJ m/mês);

$G$ - Fluxo de calor no solo, (MJ m/mês);

$\gamma$ - constante psicrométrica, $\left(\mathrm{kPa} /{ }^{\circ} \mathrm{C}\right)$;

$u_{2}$ - velocidade do vento a $2 \mathrm{~m},(\mathrm{~m} / \mathrm{s})$;

$E_{s}$ - pressão de saturação do vapor d'água atmosférico, $(\mathrm{kPa})$;

$E_{a}$ - pressão atual do vapor d'água atmosférico, $(\mathrm{kPa})$;

$T_{a}$ - temperatura média do ar, $\left({ }^{\circ} \mathrm{C}\right)$;

$\Delta$ é a tangente à curva da pressão de saturação do vapor d'água $\left(\mathrm{kPa} /{ }^{\circ} \mathrm{C}\right)$ e

$\gamma$ é o coeficiente psicrométrico $\left(0,0666 /{ }^{\circ} \mathrm{C}\right)$.

\section{Modelo evapotranspirativo Thornthwaite (1948)}

$\mathrm{ETo}($ Thor $)=16\left(10 \mathrm{~T}_{\mathrm{i}} / \mathrm{I}\right)^{\mathrm{a}}, 0^{\circ} \mathrm{C} \leq \mathrm{T}_{\mathrm{i}} \leq 26^{\circ} \mathrm{C}$

$\mathrm{ETo}_{(\text {Thor })}=-415,85+32,24 \mathrm{~T}_{\mathrm{i}}-0,43 \mathrm{~T}_{\mathrm{i}}^{2}, \quad \mathrm{~T}_{\mathrm{i}}>26^{\circ} \mathrm{C}$

Onde:

ETo(Thor) evapotranspiração de referência (mm mês $\left.{ }^{-1}\right)$;

Ti temperatura do ar média mensal $\left({ }^{\circ} \mathrm{C}\right)$; e 
I índice térmico do regime climático local computado por:

12

$$
\mathrm{I}=\Sigma\left(0,2 \mathrm{~T}_{\mathrm{i}}^{1,514}, \quad \mathrm{~T} \geq 0^{\circ} \mathrm{C}\right.
$$

$\mathrm{N}=1$

$$
A=6,75 \cdot 10^{-7} I^{3}-7,71 \cdot 10^{-5} I^{2}+1,7912 \cdot 10^{-2} I+0,49239
$$

Em que,

i meses do ano (i=1, janeiro; ...; i=12, dezembro).

As Equações 2 e 3 fornecem a evapotranspiração para a condição padrão de meses com 30 dias e dias com 12 horas de brilho solar. Para obter a evapotranspiração diária, multiplica-se o valor obtido através das Equações 2 ou 3 pelo seguinte fator de correção (Pereira et al., 1997):

$$
\mathrm{C}=\frac{\mathrm{N}}{12} \cdot \frac{1}{30}
$$

Onde:

$\mathrm{N}$ fotoperíodo (h) de determinado dia.

O fotoperíodo é calculado em função da latitude do local e da declinação solar, através da seguinte equação (Varejão-Silva, 2006):

$$
\mathrm{N}=\frac{2}{15} \omega_{\mathrm{s}}
$$

Onde:

$\mathrm{N}$ é o fotoperíodo (h) e

$\omega s$ é o ângulo horário relativo ao nascer ou ao pôr do sol.

\section{Modelo evapotranspirativo Hargreaves-Samani}

Estima a $\mathrm{ETo}_{(\text {Hars })}$ em mm/mês. Este método é utilizado na ausência de dados de radiação solar, umidade relativa do ar e velocidade do vento expressos pela seguinte Equação.

$\mathrm{ETo}_{(\mathrm{HarS})}=0,408 * 0,0023\left(\mathrm{~T}_{\text {med }}+17,8\right) *\left(\mathrm{~T}_{\max }-\mathrm{T}_{\operatorname{mim}}\right)^{0,5} \cdot \mathrm{Ra}$

$$
\mathrm{ETo}_{\text {(HS) }}=0,0023 \cdot \mathrm{R}_{\mathrm{T}} \cdot \sqrt{\mathrm{T}_{\max }-\mathrm{T}_{\min }} \cdot(\mathrm{T}+17,8)
$$

em que,

$\mathrm{ETo}_{\text {(Hars) }}$ é a evapotranspiração de referência (mm mês) estimada pelo método de Hargreaves- Samani (1985);

RT é a radiação solar extraterrestre (mm/mês);

$\mathrm{T}_{\text {med }}=$ Temperatura média $\left({ }^{\circ} \mathrm{C}\right)$; 
$\mathrm{T}_{\max }=$ Temperatura máxima $\left({ }^{\circ} \mathrm{C}\right)$;

$\mathrm{T}_{\text {mim }}=$ Temperatura mínima $\left({ }^{\circ} \mathrm{C}\right)$.

\section{Modelo evapotranspirativo Camargo}

A estimativa da $\mathrm{ETo}_{\text {(ca) }}$ foi calculada levando em consideração a equação

$\mathrm{ETo}(\mathrm{ca})=\mathrm{R}_{\mathrm{T}} . \mathrm{T}, \mathrm{K}_{\mathrm{f}} . \mathrm{ND}$

Onde:

$\mathrm{ETo}_{(\mathrm{ca})}$ evapotranspiração de referência (mm/mês) estimada pelo método de Camargo (1971);

RT radiação solar extraterrestre ( $\mathrm{mm} / \mathrm{mês}$ da evapotranspiração equivalente);

ND número de dias do período analisado;

T temperatura do ar média $\left({ }^{\circ} \mathrm{C}\right)$ e

$\mathrm{kf}$ fator de ajuste que varia com a temperatura do ar média anual do local $\left(\mathrm{kf}=0,01\right.$ para $\mathrm{T}<23{ }^{\circ} \mathrm{C} ; \mathrm{kf}=0,0105$, para $\mathrm{T}=24{ }^{\circ} \mathrm{C}$; $\mathrm{kf}=0,011$, para $\mathrm{T}=25^{\circ} \mathrm{C} ; \mathrm{kf}=0,0115$, para $\mathrm{T}=26{ }^{\circ} \mathrm{C} ; \mathrm{e} \mathrm{kf}=0,012$, para $\mathrm{T}>26^{\circ} \mathrm{C}$ ).

\section{Resultados e Discussões}

Lunardi e Rebaiolli (2013) afirmaram que um bom planejamento seguido do aproveitamento d'água na produção agrícola, assim como o desenvolvimento de metodologias que permitam estimar o correto volume d'água a ser aplicado, é de grande importância econômica às disponibilidades hídricas. Por tanto discutiremos os comparativos dos modelos evapotranspirativo de Hargreaves, Hargreaves-Samani, Camargo e Thorntwaite com o método padrão de Penman-Monteih para o período de 1963-2020 em Amparo de São Francisco - Sergipe, nas seguintes Figuras (2 a 6).

Na Figura 2 observam-se as variabilidades evapotranspirativas dos métodos Penman-Monteit e Thornthwaite e seu percentual entre os referidos modelos para o período de 1963-2019 em Amparo de São Francisco - Sergipe.

No método Penman-Monteith registra-se média de 2137,7 mm, seu máximo absoluto ocorreu no ano de 2015 com 2170,6 mm e seu mínimo em 1964 com 1955,1 mm. O modelo Thornthwaite registou média de 1553,0 mm e seus máximos e mínimos evatranspirativos foram de 1653,7 mm e 1435,7. Destaca-se que no método Penman-Monteith ocorreram subestimação dos valores da ETo e o modelo Thornthwaite registrou valores coerentes a serem utilizados nos projetos agroclimáticos da região. 
Figura 2. Evapotranspiração potencial pelo método Penman-Monteith, Thornthwaite e seu percentual entre os referidos modelos para o período de 1963-2020 em Amparo de São Francisco - Sergipe.

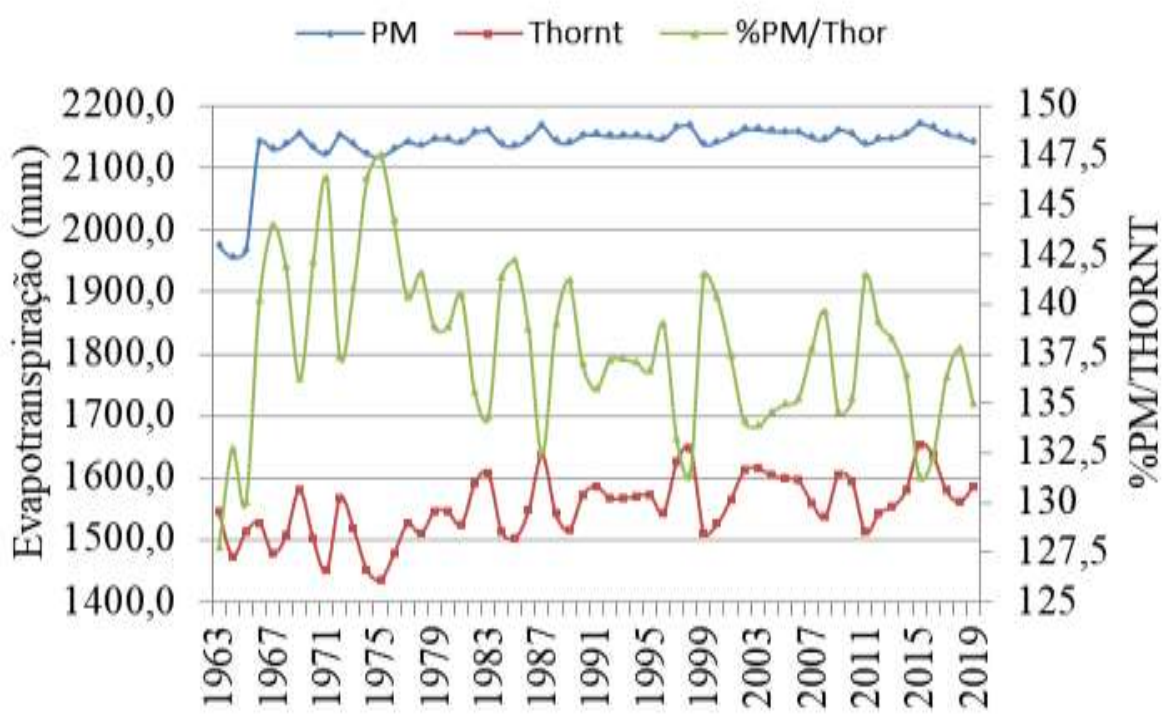

Fonte: França (2021).

Na Figura 3. Tem-se as variabilidades da evapotranspiração potencial pelo método Penman-Monteith e Hargreaves e seu percentual entre os referidos modelos para o período de 1963-2020 em Amparo de São Francisco - Sergipe.

Nos modelos Penman-Monteith os seus valores estão subestimados em relações ao modelo de Hargreaves. O Modelo Hargreaves registra valores evapotranspirativos intermediários que podem ser utilizados para a área de estudo. Estes resultados estão de acordo com vários estudos realizados para o semiárido nordestino, conforme (Guedes Filho, Santos Junior, Costa Filho \& Campos 2011; Matos, Silva \& Medeiros 2014; Marengo, Alves, Beserra \& Lacerda 2012: Medeiros, Holanda, Saboya, Rolin Neto, Araújo \& França 2021).

Figura 3. Evapotranspiração potencial pelo método Penman-Monteith, Hargreaves e seu percentual entre os referidos modelos para o período de 1963-2020 em Amparo de São Francisco - Sergipe.

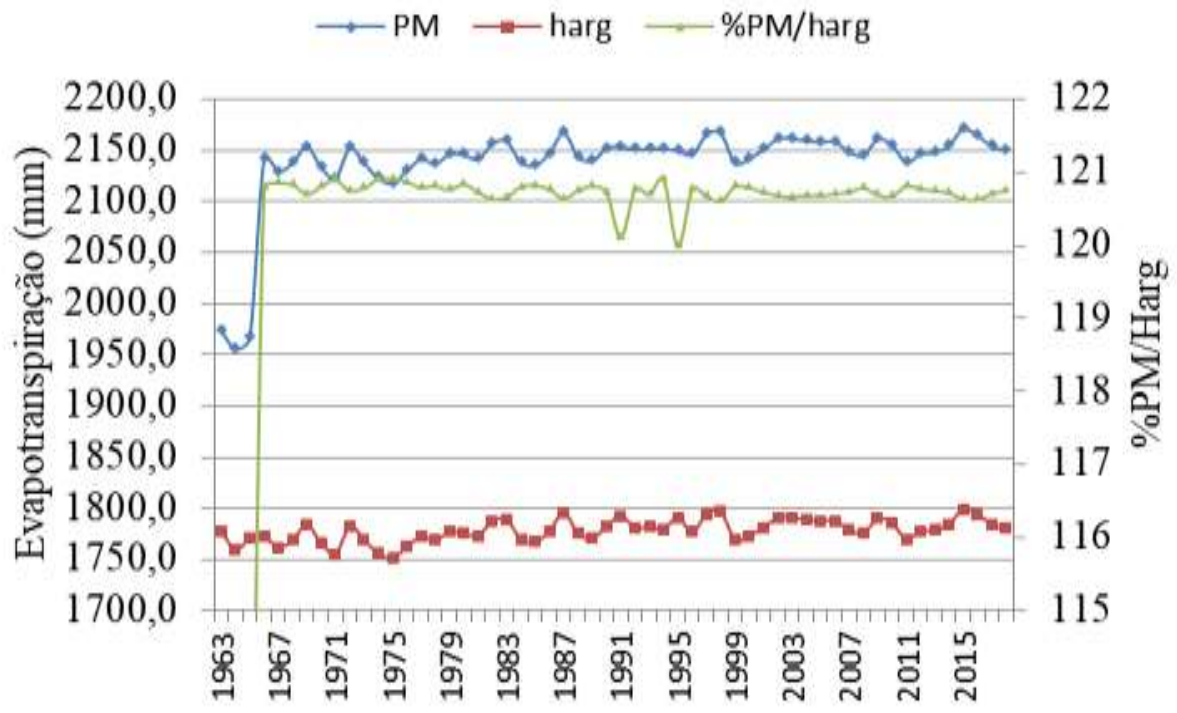

Fonte: França (2021). 
Dantas' Oliveira, Darli, Paralleti, Santos e Farias (2016) confrontou a exatidão dos métodos de Hargreaves-Samani e Camargo, em relação ao método de Penman-Monteith para as mesorregiões do estado da Paraíba, sua avaliação para os resultados, indicou que o modelo de Hargreaves-Samani não necessitou de ajustes para a região do agreste, mas o mesmo modelo não se ajustou à região da Borborema. Este resultado vem a corroborar com os discutidos neste artigo.

A evapotranspiração potencial pelo método Penman-Monteith, Hargreaves-Samani e seu percentual entre os referidos modelos, para o período de 1963-2020 em Amparo de São Francisco - Sergipe. (Figura 4).

O Hargreaves-Samani subestima seus valores quando comparados ao modelo Penman-Monteith, ambos têm seus valores subestimados. Os valores percentuais demonstram grande variabilidade registrada para os modelos.

Figura 4. Evapotranspiração potencial pelo método Penman-Monteith, Hargreaves-Samani e seu percentual entre os referidos modelos, para o período de 1963-2020 em Amparo de São Francisco - Sergipe.

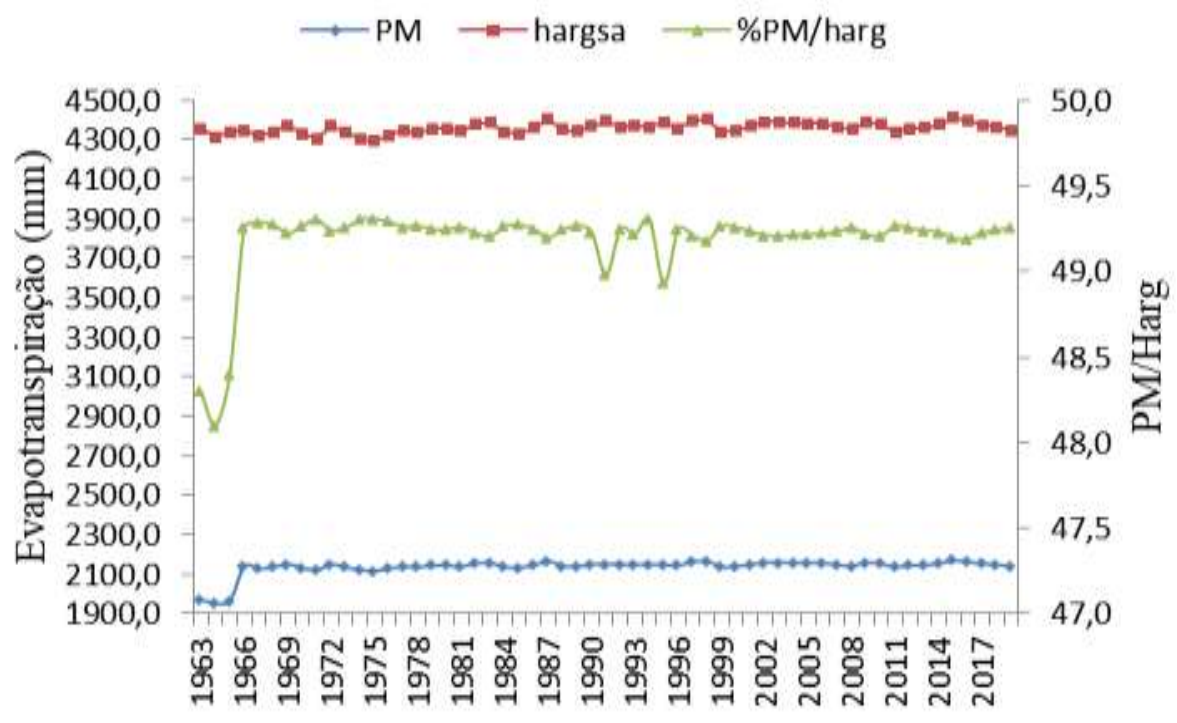

Fonte: França (2021).

Na Figura 5 Observam as flutuações da ETo pelo método Penman-Monteith e Camargo e seu percentual entre os referidos modelos, no período de 1963-2019 em Amparo de São Francisco - Sergipe. No método Penman-Monteith registra-se média de 21377,7 mm, seu máximo absoluto ocorreu no ano de 2015 com 2170,6 mm e seu mínimo em 1964 com 1955,1 mm. No método Camargo a média registrada é de 1665,8 mm e seus máximos e mínimos absoluto ocorreram em 1987 e 1975 com 1735,9 mm e $1566,6 \mathrm{~mm}$ respectivamente.

Os percentuais comparativos entre os dois métodos demonstram grandes flutuabilidades com média de 128,4 mm e seus máximos e mínimos ocorrendo entre 135,2 mm e 117,8 (Figura 5). O método Penman-Monteith subestima os valores da ETO e o modelo Camargo registram valores mais coerentes para serem aplicados nos estudos agroclimáticos da região em estudo. 
Figura 5. Evapotranspiração potencial pelo método Penman-Monteith e Camargo e seu percentual entre os referidos modelos, para o período de 1963-2020 em Amparo de São Francisco - Sergipe.

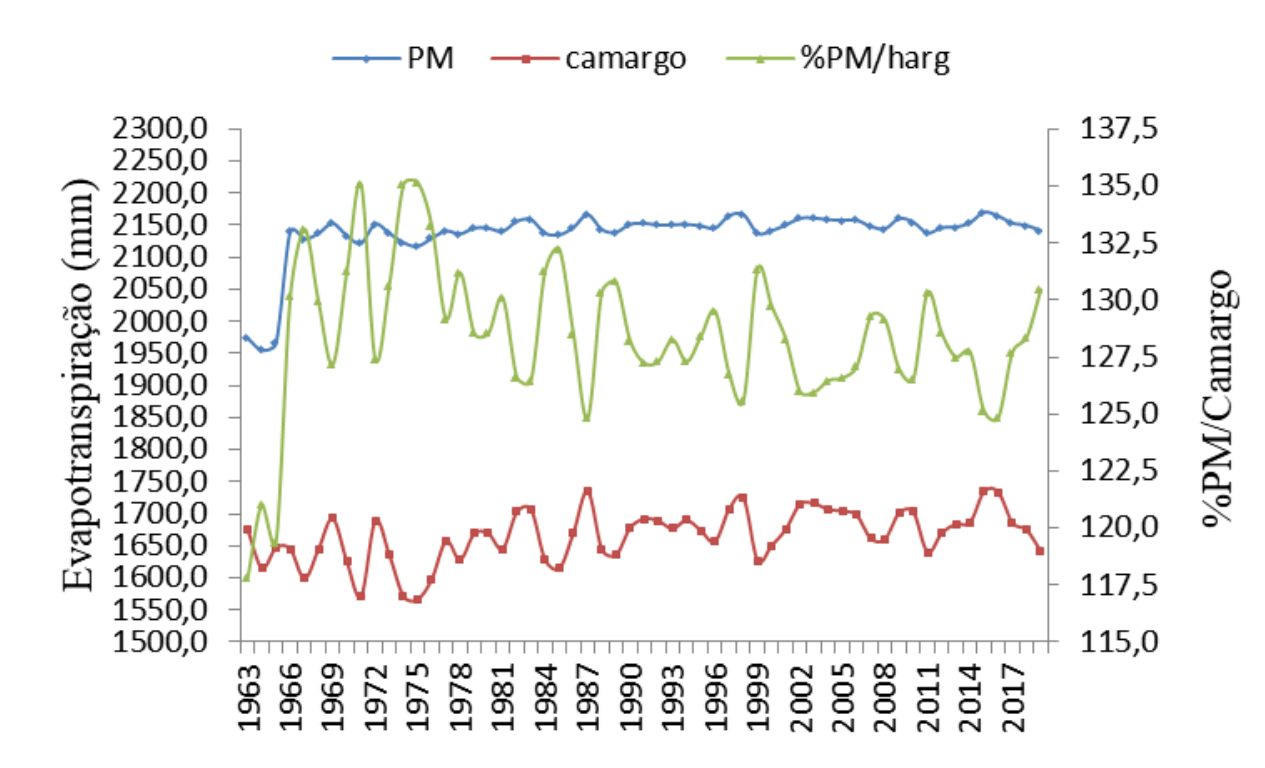

Fonte: França (2021).

Na Figura 6 observam-se os comparativos da evapotranspiração potencial pelo método de Thornthwaite, Camargo, Hargreaves-Samani, Hargreaves, Penman-Monteith e os índices pluviais para o período de 1963-2019 em Amparo de São Francisco - Sergipe.

Realizando um comparativo evapotranspirativos entre os modelos estudados, observam-se que Hargreaves-Samani subestima seus valore quando comparados aos demais e para o modelo Penman-Monteith os seus valores estão subestimados em relações aos outros três estudados. O Modelo Hargreaves registra valores evapotranspirativos superiores aos modelos de Camargo e Thornthwaite. Os modelos de Thornthwaite e Camargo registraram índices evapotranspirativos praticamente similares e que deverão ser aplicados com maiores confiabilidades nas aplicações agrícolas do município.

Alencar, Sediyana e Montvani (2015) observaram uma superestimava da evapotranspiração mensal pelo método da estimativa de Penman-Monteith e atribui o resultado a velocidade do vento, o que vem a corroborar com os resultados em discussões. 
Figura 6. Comparativos da evapotranspiração potencial pelo método de Thornthwaite, Camargo, Hargreaves-Samani, Hargreaves, Penman-Monteith e os índices pluviais para o período de 1963-2020 em Amparo de São Francisco - Sergipe.

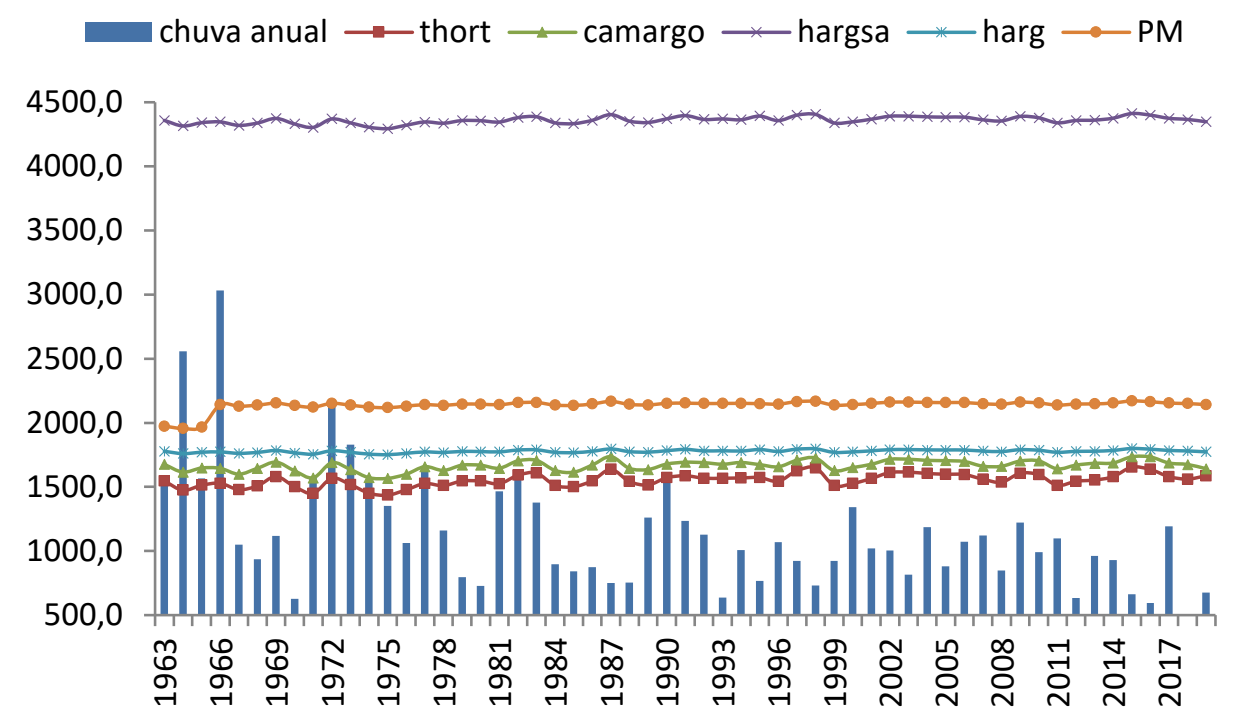

Fonte: França (2021).

Na Tabela 1 têm-se as demonstrações dos parâmetros estatísticos da precipitação (Prec) e para os modelos evapotranspirativos de Thornthwaite, Camargo, Hargreaves-Samani, Hargreaves, Penman-Monteith e os índices pluviais para o período de 1963-20120 em Amparo de São Francisco - Sergipe.

As médias foram subestimadas para os modelos Hargreaves-Samani e Penman-Monteith. A mediana se distancia dos valores médios e não são representativos no estudo, os desvios padrões dos modelos são de baixa significância e os valores, exceto o desvio padrão da pluviometria que é altamente elevado para a área de estudo. Os coeficientes de variância não são representativos para os valores pluviais e dos modelos. Os valores máximos e mínimos absolutos foram ocasionados pelos sistemas predominantes nas escalas meso e micro e as suas contribuições local e regional, estudos com similaridades de dados foram encontrados em Marengo, Santos, Saulo e Nicolini (2004). 
Tabela 1. Parâmetros estatísticos da precipitação (Prec) e para os modelos evapotranspirativos de Thornthwaite, Camargo, Hargreaves-Samani, Hargreaves, Penman-Monteith e os índices pluviais para o período de 1963-2020 em Amparo de São Francisco - Sergipe.

\begin{tabular}{l|c|c|c|c|c|c}
\hline \multicolumn{1}{c|}{ Parâmetros } & $\begin{array}{c}\text { Precipitação } \\
\text { Anual }\end{array}$ & Thornthwaite & Camargo & Hargsa & Harg & PM \\
\hline Média & 1138,2 & 1553,0 & 1665,8 & 4360,2 & 1778,2 & 2137,7 \\
\hline Mediana & 1129,0 & 1567,9 & 1689,0 & 4366,2 & 1780,7 & 2150,4 \\
\hline Desvio padrão & 470,7 & 50,4 & 40,7 & 27,1 & 11,1 & 42,6 \\
\hline Coeficiente variância & 0,414 & 0,032 & 0,024 & 0,006 & 0,006 & 0,020 \\
\hline Máximo absoluto & 3032,8 & 1653,6 & 1735,9 & 4412,2 & 1799,4 & 2170,5 \\
\hline Mínimo absoluto & 498,8 & 1435,7 & 1566,5 & 4294,2 & 1751,3 & 1955,1 \\
\hline
\end{tabular}

Fonte: França (2021).

No período de inverno, a diferença foi menor visto que nessa estação registra-se influência da radiação, temperatura e da velocidade do vento, que são variáveis importantes nos poderes evapotranspirativos e evaporativos da água. Resultado semelhante foram encontrados nos estudos dos autores (Hallal, Schoffel, Brixner \& Raduns 2013; Muniz. Mendonça, Esteves, Liusada (2014); Cardoso et al. (2014). Em estudo da variabilidade intra e interanual da bacia hidrográfica estudada, Piazza, Pinheiro, Kaufmann, Torres, Venzon, Gotsrdo, Baptista e Knaesel (2017) também encontrou valores de ETp maiores no verão (janeiro a março) e menores durante o inverno (junho a agosto).

\section{Conclusões}

Dentre os métodos baseados na temperatura, o método de Hargreaves-Samani é o que apresentou subestimativas da evapotranspiração de referência em relação aos demais modelos. As mudanças e as adaptações dos usos da terra, tais como agricultura, pastagem e reflorestamento, causam alterações na dinâmica da água da bacia hidrográfica. O método de Penman Monteith superestima os valores da ETo mensal em todos os usos da terra estudados. Este comportamento pode estar atrelado a radiação solar e a temperatura do ar que são os fatores determinantes na estimativa da evapotranspiração pelo método de PenmanMonteith.

Observou-se melhor adaptação do modelo de Penman-Monteith e Thornthwaite na estimativa da ETo onde seus resultados mostraram-se adaptação para a localidade estudada. Aconselha-se a avaliação de outros modelos para que possam proporcionar resultados mais apropriados as mesmas. Afirma-se que os modelos de Penman-Monteith e Thornthwaite são as melhores alternativas para o uso da evapotranspiração em Amparo de São Francisco - Sergipe.

Em Amparo de São Francisco, posicionado ao nordeste do estado, não existem trabalhos sobre o uso racional d'água. A avaliação de métodos empíricos de estimativa da evapotranspiração como Hargreaves, Hargreaves-Samani, Camargo e Thorntwaite, comparados com o método padrão de Penman-Monteih, poderão auxiliar no planejamento e gerenciamento de irrigação na região, principalmente por parte dos pequenos irrigantes que não têm acesso aos dados meteorológicos.

Na falta de elementos meteorológicos necessários para determinar a evapotranspiração, a equação de Thornthwaite pode ser utilizada com razoável precisão para a estimativa da evapotranspiração na área estudada. 


\section{Referências}

Alencar, L. P., Sediyama, G. C. \& Montovani, E. C. (2015). Estimativa da evapotranspiração de referência (ETo padrão FAO) para Minas Gerais na ausência de alguns dados climáticos. Engenharia Agrícola, 35(1), 39- 50. http://doi.org/10.1590/1809-4430

Allen, R. G., Pereira, L. S., Raes, D. \& Smith, M. (1998). Crop evapotranspiration: guidelines of computing crop water requirements. FAO. Irrigation and Drainage Paper, 56, 300,

Alvares, C. A., Stape, J. L., Sentelhas, P. C., Gonçalves, J. L. M. \& Sparovek, G. (2014). Climate classification map for Brazil. Meteorologische Zeitschrift 22, 711728.

Barros, R., Souza, A. P., Fonseca, D. C. \& Silva, L. B. D. (2009). Avaliação da evapotranspiração de referência na Região de Seropédica, Rio de Janeiro, utilizando lisímetro de pesagem e modelos matemáticos. Revista Brasileira de Ciências Agrárias, 4(2), 198-203. http://doi.org/10.5039/agraria.v4i2a13.

Barros, A. H. C., Araújo Filho, J. C., Silva, A. B., \& Santiago. G. A. C. F. (2012). Climatologia do Estado de Alagoas. Boletim de Pesquisa e Desenvolvimento n.211. Embrapa Solos, 32p.

Borges, A. C. \& Mendiondo, E. M. (2007). Comparação entre equações empíricas para estimativa da evapotranspiração de referência na bacia do rio Jacupiranga. Revista Brasileira de Engenharia Agrícola e Ambiental, 11(3), 293-300.

Cardoso, G.M., \& Justino, F. Simulação dos componentes da evapotranspiração sob 29(1), 85-95, 2014. http://doi.org/10.1590/S0102-77862014000100009

Camargo, A. P. \& Sentelhas, P. C. (1997). Avaliação do desempenho de diferentes métodos de estimativa da evapotranspiração potencial no estado de São Paulo, Brasil. Revista Brasileira de Agrometeorologia, 5(1), 89-97.

Camargo, A. P. \& Sentelhas, P. C. (1999). Ajuste da equação de Thornthwaite para estimar a evapotranspiração potencial em climas áridos e superúmidos, com base na amplitude térmica diária. Revista Brasileira de Agrometeorologia, 7(2), 251-257.

Cavalcanti, E. P., Silva, P. R., \& Sousa, F. A. S. Programa computacional para a estimativa da temperatura do ar para a região Nordeste do Brasil. Revista Brasileira de Engenharia Agrícola e Ambiental, 10 (1), 140-147. 2006.

Cavalcanti, E. P., \& Silva, E. D. Estimativa da temperatura do ar em função das coordenadas locais. In: Congresso Brasileiro de Meteorologia, 8, 1994. Belo Horizonte, Anais..., SBMET, 1, 154-157.

Costa, M. N. M., Medeiros, R. M., \& Gomes Filho, M. F. (2015). Variabilidade da evaporação no tanque classe "A" entre o período de 1976 a 2011 e suas comparações com as três décadas e meia e com o ano de 2011 no município de Teresina, Piauí. $7^{\circ}$ Workshop de Mudanças Climáticas do Estado de Pernambuco e $4^{\circ}$ Workshop Internacional sobre Mudanças Climáticas e Biodiversidade. Recife-PE, CD RON. 107-123.

Cunha, F. F., Magalhães, F. F., \& Castro, M. A. (2013). Métodos para estimativa da evapotranspiração de referência para Chapadão do Sul-MS. Engenharia na Agricultura, 21(2), 159-172, 2013. https://doi.org/10.13083/reveng.v21i2.346

Dantas, G. F., Oliveira, M. R., Dalri, A. B., Palaretti, L. F., Santos, M. G., \& Faria, R. T. T. (2016). Desempenho de métodos na estimativa de evapotranspiração de referência para o estado da Paraíba, Brasil. Irriga, 21(3), 481-490.

Doorenbos, J., \& Pruitt, W. O. (1977). Guidelines for predicting crop water requirements. FAO, 1977.

Gebler, S., Franssen, H. J. H., Pütz, T., Post, H., Schmidt, M., \& Vereecken, H. (2015). Actual evapotranspiration and precipitation measured by lysimeters: a comparison with eddy covariance and tipping bucket. Hydrology and Earth System Sciences, 19, 2145-2161. https://doi.org/10.5194/hess-19-2145-2015

Goulden, M. L., Litvak, M. \& Miller, S. D. (2007). Factors that control Typha marsh evapotranspiration Aquatic. Botany, 86, 97106http://doi.org/10.1016/j.aquabot.2006.09.005.

Guedes Filho, D. H., Santos Júnior, J. A., Costa Filho, J. F. \& Campos, B. (2011). Estimativa da evapotranspiração de referência para a cidade de Areia, Paraíba. Revista Brasileira de Agricultura Irrigada, 5(1), 37-47.

Hallal, M. O. C., Schoffel, E. R., Brixner, G. F., \& Raduns, A. L. (2013). Estimativa da evapotranspiração de referência e sua relação com a precipitação ocorrida na região de Pelotas-RS. Irriga, 18(1),.85-98. http://doi.org/10.15809/irriga.2013v18n1p85

Hargreaves, G. H. (1977). World water for agriculture. Utah State University,

Hargreaves, G. H. \& Samani, Z. (1985). Reference crop evapotranspiration from temperature. Journal of Applied Engineering in Agriculture, St Joseph, 1, 96-99.

Lunardi, J., Rabaiolli, J. A. (2013). Valorização e preservação dos recursos hídricos na busca pelo desenvolvimento rural sustentável. Revista OKARA: Geografia em debate, 7, 44-62.

Marengo, J. A., Soares, W. R., Saulo, C. \& Nicolini, M. (2004). Climatology of the low-level Jet East of the Andes as Derived from NCEP-NCAR Reanalyses: Characteristics and Temporal Variability. Journal of Climate, 17, 2261-2280.

Marengo, J. A., Alves, L. M., Beserra, E. A., \& Lacerda, F. F. (2012). Variabilidade e mudanças climáticas no semiárido brasileiro. Recursos hídricos em regiões áridas e semiáridas. 303-422.

Matos, R. M., Silva, J. A. S., \& Medeiros, R. M. (2014). Aptidão climática para a cultura do feijão caupi do município de Barbalha - CE. Revista Brasileira de Agricultura Irrigada, 8(6), 422-431. 
Medeiros, R. M., Holanda, R. M., Saboya, L. M. F., Rolim Neto, F. C., Araújo, W. P., França, M. (2021). Espacialização pelo método da Krigagem nas variabilidades pluvial, evapotranspiração e evaporação no Estado do Pernambuco - Brasil. Research, Society and Development, 10, n. 2, e51010212758, 2021 (CC BY 4.0)| ISSN 2525-3409 | http://dx.doi.org/10.33448/rsd-v10i2.12758

Medeiros, R. M. (2020). Estudo Agrometeorológico para o Estado do Sergipe. p.175.

Medeiros, R. M., Holanda, R. M., Viana, M. A., Silva, P. (2018). CLIMATE Classification in Köppen model for the State of Pernambuco - Brazil. Revista de Geografia (Recife). 35, $219-234$.

Muniz, R. A., Souza, E. F., Mendonça, J. C., Esteves, B. S., Liusada, L. L. (2014). Balanço de energia e evapotranspiração do capim Moçamba sob sistema de pastejo rotacionado. Revista Brasileira de Meteorologia, 29,.47-54, 2014. http://doi.org/10.1590/S0102- 77862014000100005

Penman, H. L. (1948). Natural evaporation from open water, bare soil, and grass. Proceedings of the Royal Society, 193(1), 20-146. http://doi.org/10.1098/rspa.1948.0037

Pereira, A. R., Villa Nova, N. A., Sediyama, G.C. Evapo(transpi)ração. Piracicaba: FEALQ, 1997. 183p.

Pereira, A. R., Pruitt, W. O. (2004). Adaptation of the Thornthwaite scheme for estimating daily reference evapotranspiration. Agricultural Water Management, Amsterdam, Netherlands, 66, 251-257.

Piazza, G. A., Pinheiro, A., Kaufmann, , Torres, E., Venzon, P. T., Gotardo, R., Baptista, G. C.Z., Knaesel, K. M. (2017). Histerese intranual das variáveis hidroclimáticas da Bacia do Ribeirão Concórdia, Santa Catarina, Sul do Brasil. In: Simpósio Brasileiro de Recursos Hídricos, 20. Anais. Florianópolis: UFSC.

Priestley, C. H. B., Taylor, R. J. (1972). On the assessment of surface heart flux and evaporation using large-scale parameters. Monthly Weather Review, 100, 8192, https://doi.org/10.1175/1520-0493(1972)1002.3.CO,2

Santos, A. A. R., Lyra, G. B., Lima, E. P., Souza, J. L., Delgado, R. C. C. (2016). Evapotranspiração de referência em função dos extremos da temperatura do ar no estado do Rio de Janeiro. Irriga, 21, 449-465, 2016.

Santos, R. D. S., Souza, M. H. C., Bispo, R. C., Ventura, K. M., Bassoi, L. H. H. (2017). Comparação entre métodos de estimativa da evapotranspiração de referência para o município de Petrolina, PE. Irriga, 1(1), 31-39.

Schrader, F., Duner, W., Fank, J., Gebler, S., Putz, T., Hannes, M. \& Wollschlager, U. (2013). Estimating precipitation and actual evapotranspiration from precision lysimeter measurements. SciVerse Science Direct, 19, 543-552, 2013. https://doi.org/10.1016/j.proen2013.06.061

Sediyama, G. C. (1996). Evapotranspiração: Necessidade de água para plantas cultivadas. Brasília - DF: ABEAS/UF 176p. (curso de engenharia da irrigação, módulo $4)$.

Thornthwaite, C. W. (1948). Na approach toward a rational classification of climate. Geographical Review, 38 : 55-94.

Unlu, M., Kapur, B. (2010). Comparison of soybean evapotranspiration measured by weighing lysimeter and Bowen ratio-energy balance methods. African Journal of Biotechnology, 9(30),.4700-4713.

Tucci, C. E. M. (2005). Modelos hidrológicos- (2a ed.), Editora da UFRGS, 669p.

Varejão-Silva, M. A. (2006). Meteorologia e Climatologia. Versão Digital, 443p. 